\title{
Objetos de Aprendizagem para auxiliar o ensino de conceitos do Paradigma de Programação Orientada a Objetos
}

\author{
Mychelline Souto Henrique - UFPE, msh@cin.ufpe.br \\ Ayla Débora Dantas Souza Rebouças - UFPB, ayla@dce.ufpb.br
}

\begin{abstract}
Resumo: Os altos índices de reprovação nas disciplinas de programação é uma problemática conhecida pela comunidade de informática na educação. Vários estudos mostram a eficácia do uso de Objetos de Aprendizagem (OAs) no processo de ensino, e essa parece ser uma alternativa viável para tornar as aulas mais atrativas, e diminuir o nível de abstração advindos da programação. Este artigo apresenta os índices de reprovação da disciplina de Programação Orientada a Objetos (OO) da UFPB - Campus IV durante oito semestres e que se mostraram na maioria dos casos superiores a 50\%. Para identificar como OAs podem ajudar a minimizar essa problemática, foi realizado um survey com alunos para identificar os conceitos de OO considerados de difícil compreensão e também um mapeamento sistemático para identificar OAs que auxiliem o ensino dos conceitos de OO considerados difíceis pelos alunos. Acredita-se que este estudo auxiliará professores na escolha de OAs a explorar por conteúdo e dará uma ideia dos OAs apontados pela literatura. Além disso, servirá como base para o projeto e desenvolvimento de novos OAs para apoiar o processo de ensino-aprendizagem de POO.
\end{abstract}

Palavras-chave: objetos de aprendizagem, programação orientada a objetos, mapeamento sistemático

\section{Learning Objects to aid the teaching process of Objetct-Oriented Programming}

\begin{abstract}
The high indexes of students that flunk in the programming disciplines is a problem known by the informatics in education community. Several studies show the effectiveness of the use of Learning Objects (LOs) in the teaching process, and they seem to be a viable alternative to make classes more attractive, and to reduce the level of abstraction of programming. This article presents the failure rates in the Object-Oriented (OO) programming discipline of UFPB Campus IV in eight semesters, which showed to be over 50\% in most of the cases. In order to identify how OAs can minimize that reality, a survey with students was performed to identify the concepts of $\mathrm{OO}$ considered difficult by students and we have performed a mapping study to identify LOs to aid the teaching of these concepts considered difficult by students. We believe this work will aid OO teachers to choose LOs to explore in their classes by subject and will give them an idea of the LOs discussed in the literature. Besides, this study can support the project and development of new LOs to aid the teaching/learning process of OO programming.
\end{abstract}

Keywords: learning objects, object-oriented programming, mapping study

\section{Introdução}

Com o passar do tempo, os métodos de ensino foram evoluindo. Além disso, as informações ficaram mais acessíveis através da facilidade de acesso à Internet, e novos recursos trazidos pelas tecnologias adentraram no ambiente escolar para auxiliar o professor no processo de ensino/aprendizagem. Esses recursos possuem um grande potencial pedagógico, pois eles tornam as aulas mais atrativas e motivadoras e fazem a educação seguir os caminhos traçados pela tecnologia (Flôres e Tarouco, 2008).

Observa-se, portanto, que é importante que o professor, seja ele de qualquer área, aperfeiçoe sua metodologia de ensino e planeje suas aulas utilizando recursos 
tecnológicos criados com propósito educativo. Alguns desses recursos são conhecidos como Objetos de Aprendizagem (OAs). Neste trabalho será considerada a definição de Wiley (2000), que afirma que um objeto de aprendizagem é uma entidade que pode ou não ser digital, e que pode ser usada, reusada ou referenciada durante o ensino com suporte tecnológico.

Os professores que lecionam disciplinas de programação em cursos da área de informática ou de engenharia convivem com uma grande dificuldade vivenciada por vários alunos para aprender os conceitos básicos para construir um programa de computador. Em paralelo, sabe-se de uma forte demanda do mercado por profissionais desenvolvedores de software em todo o mundo ${ }^{1}$, o que torna o problema da dificuldade dos alunos bem relevante. O trabalho de Raabe e Silva (2005) identificou vários fatores que podem influenciar no processo de ensino/aprendizagem de programação. Para isso, os autores realizaram uma análise sistemática nas disciplinas de algoritmos e programação durante nove semestres, e dividiram as causas em três grupos distintos: (i) problemas de natureza didática; (ii) problemas de natureza cognitiva; e (iii) problemas de natureza afetiva. Em relação à natureza didática, que é o foco deste trabalho, alguns problemas são: o grande número de alunos; o ambiente de realização das provas; a ausência de bons materiais. Sendo assim, percebe-se uma necessidade de identificação de OAs existentes de modo sistemático, observando também os conteúdos que buscam apoiar.

Este trabalho surgiu da necessidade de auxiliar alunos e professores no processo de ensino/aprendizagem na disciplina de linguagem de programação, ofertada no $2^{\circ}$ período da UFPB- Campus IV no curso de Licenciatura em Ciência da Computação (LCC) e também no curso de Sistemas de Informação (SI). A linguagem utilizada é Java, que segue o Paradigma da Programação Orientada a Objetos (POO). O índice de reprovação na disciplina no Campus é muito alto, e essa realidade se repete a cada período.

Considerando a existência de ferramentas discutidas na literatura, foi realizado um mapeamento sistemático com o propósito de descobrir trabalhos relacionados ao uso de OAs que foram desenvolvidos para apoiar o ensino das disciplinas de programação OO. Ao final da pesquisa foi constatado que existem vários OAs citados pelos trabalhos, como: jogos, simulações, "frameworks", vídeos, animações, Sistemas tutores Inteligentes (STI), dentre outros. Para identificar os conteúdos considerados de difícil compreensão, este trabalho realizou um survey com os alunos de LCC e SI. Por fim, foi possível identificar por OA os conceitos de OO que eles cobriam.

Esse trabalho se torna importante porque aborda um problema comum, que é o alto índice de reprovação na disciplina de programação. Além disso, ele é importante para que os professores que lecionam o paradigma $\mathrm{OO}$, que se identifiquem com as dificuldades aqui relatadas, possam utilizar os OAs identificados no mapeamento sistemático em suas aulas.

Este artigo está organizado da seguinte forma: a Seção 2 discute os trabalhos relacionados; a Seção 3 mostra os índices de aprovações e reprovações de alunos de LCC e SI na disciplina introdutória de OO; a Seção 4 mostra a metodologia utilizada por esse trabalho: survey e mapeamento sistemático na literatura; a Seção 5 mostra os resultados desta pesquisa. Por fim, a Seção 6 apresenta as conclusões e trabalhos futuros.

\footnotetext{
${ }^{1}$ http://exame.abril.com.br/carreira/noticias/os-profissionais-mais-buscados-no-brasil-e-em-35-paises
} 


\section{Trabalhos relacionados}

Alguns trabalhos da literatura apresentam revisões e mapeamentos sistemáticos sobre softwares educacionais (Ex: jogos, simulações) e ferramentas que auxiliam o processo de ensino da programação. Porém, percebe-se que o termo Objeto de Aprendizagem não foi utilizado em alguns casos, embora tais recursos tenham sido criados para auxiliar o processo de ensino e possam ser considerados como tais e alguns trabalhos tratam de ferramentas para programação em geral. A Tabela 1 mostra esses trabalhos e algumas ferramentas utilizadas para auxiliar no ensino de programação.

Tabela 1 - Estudos secundários sobre o uso de ferramentas para auxiliar o ensino de programação

\begin{tabular}{|c|c|}
\hline Estudos secundários & $\begin{array}{l}\text { Ferramentas utilizadas no ensino de } \\
\text { programação }\end{array}$ \\
\hline $\begin{array}{l}\text { Marcolino. A; Barbosa. E.F. Softwares Educacionais } \\
\text { para o Ensino de Programação: Um Mapeamento } \\
\text { Sistemático. SBIE (2015) }\end{array}$ & $\begin{array}{l}\text { CABLE (Cognitive Apprenticeship-Based Learning } \\
\text { Environment); SimplifIDE; Alice; Scratch; Greenfoot; } \\
\text { 3D Avatar Programming System; Storytelling Alice; } \\
\text { CodeSpells; Modkit; LilyPad ProtoSnap board. }\end{array}$ \\
\hline $\begin{array}{l}\text { Silva. T.R; Medeiros. T. J; Aranha. E. H. S. Jogos } \\
\text { Digitais para Ensino e Aprendizagem de Programação: } \\
\text { uma Revisão Sistemática da Literatura. SBIE (2014) }\end{array}$ & $\begin{array}{l}\text { Greenfoot; Alice; Scratch; Blocky; Game Maker; } \\
\text { Lego Mindstorms; LighBot; Gidget(jogo); }\end{array}$ \\
\hline $\begin{array}{l}\text { Medeiros. T. J; Silva. T.R; Aranha. E. H. S. Ensino } \\
\text { de programação utilizando jogos digitais: uma } \\
\text { revisão sistemática da literatura. RENOTE (2013) }\end{array}$ & $\begin{array}{l}\text { PyGame; RoboMind; Lego Mindstorms; Takkou; } \\
\text { Scratch; Alice; iVprog; Escracho; Kodu; Game } \\
\text { Maker; Construct } 2\end{array}$ \\
\hline $\begin{array}{l}\text { Aureliano. V. C.O; Tedesco. P. C. A. R. Ensino- } \\
\text { aprendizagem de Programação para Iniciantes: uma } \\
\text { Revisão Sistemática da Literatura focada no SBIE e } \\
\text { WIE. SBIE (2012) }\end{array}$ & $\begin{array}{l}65 \% \text { dos artigos propõem ferramentas de software, mas } \\
\text { o artigo não detalha quais são essas ferramentas. }\end{array}$ \\
\hline
\end{tabular}

As ferramentas mais utilizadas no processo de ensino de programação, segundo os trabalhos apresentados na Tabela 1 são: Alice, Scratch, Greenfoot, Game Maker e Lego Mindstorms. Nota-se, porém, uma demanda por um levantamento sistemático de OAs para apoiar no ensino de $\mathrm{OO}$ e seus conceitos e não programação em geral, como nestes trabalhos, que foi o que motivou este trabalho.

\section{3. Índices de aprovações e reprovações de alunos de LCC e SI na disciplina de POO}

Esta seção apresenta os índices de retenção na disciplina introdutória programação OO dos cursos de LCC e SI da UFPB - Campus IV. Os índices são altos e isso se repete em todos os períodos, em ambos os cursos: LCC e SI. A Figura 1 mostra os índices de aprovação e reprovação ou abandono para os alunos matriculados dos cursos de LCC e SI entre os períodos de 2009.2 até 2013.1.

Observando os dados, pode-se perceber que a quantidade de aprovados é na grande maioria das vezes menor do que a quantidade de reprovados, mesmo para diferentes professores lecionando a disciplina. 


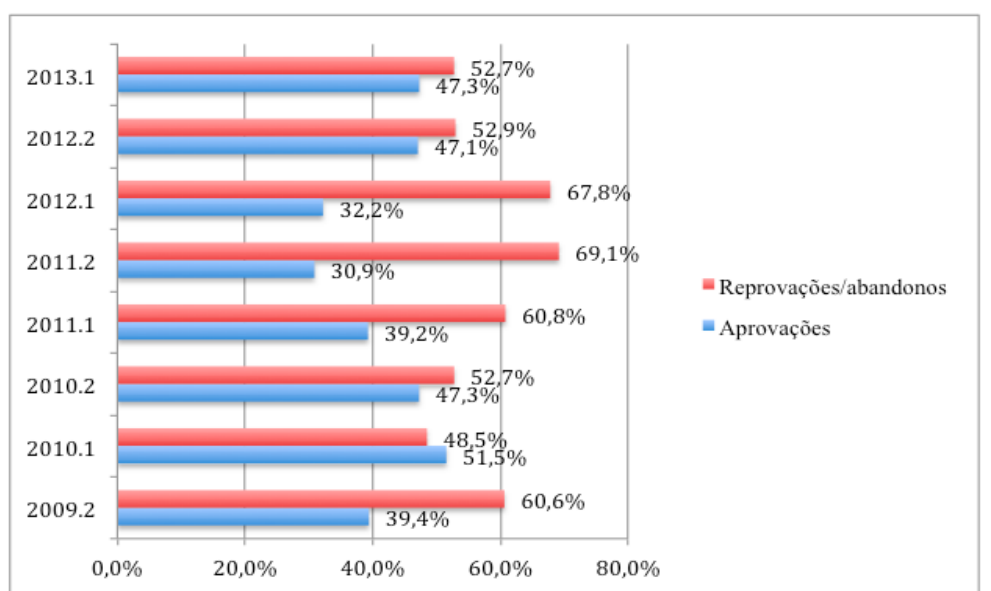

Figura 1 - Resumo dos índices de aprovações e reprovações ou abandonos na disciplina introdutória de programação OO dos cursos de LCC e SI da UFPB - Campus IV por período

A complexidade em relação aos conceitos de programação $\mathrm{OO}$ pode ser um dos fatores que leva a esses números, e esta já é discutida por vários trabalhos da área de ensino de computação e informática. Por isso, se faz necessário criar alternativas de ensino para auxiliar os alunos durante a disciplina e motivá-los mais, já que os índices de abandono (trancamentos ou reprovações por falta) na disciplina são bem altos, conforme ilustra a Figura 2.

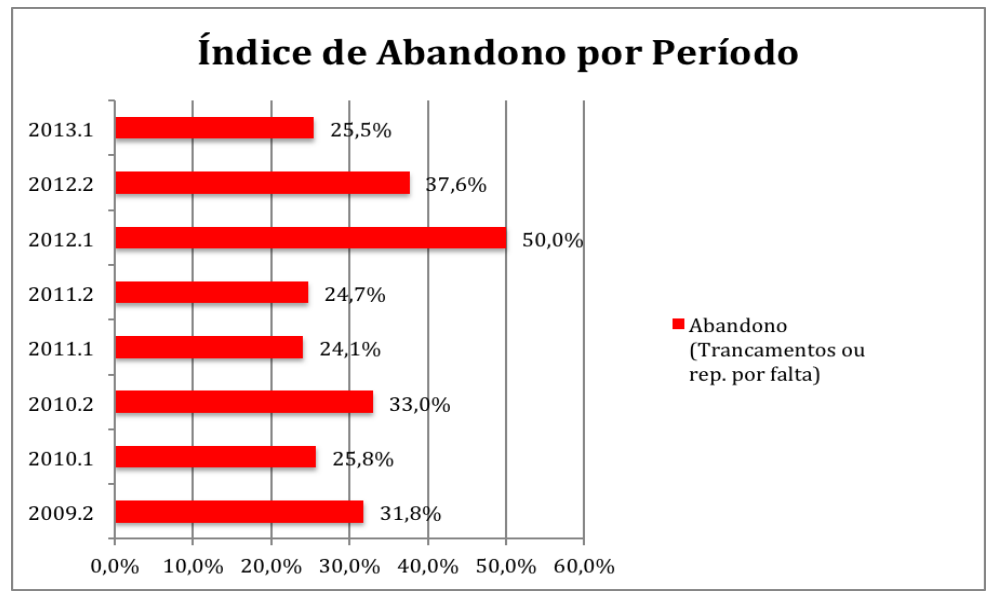

Figura 2 - Resumo dos índices de abandono na disciplina introdutória de programação $\mathrm{OO}$ dos cursos de LCC e SI da UFPB - Campus IV por período

É perceptível que muitos alunos acabam abandonando a disciplina, o que é refletido nos números de reprovados por falta ou trancamentos, que são apenas parte das reprovações/abandonos. Esses percentuais de abandono foram superiores a $24 \% \mathrm{em}$ todos esses períodos, chegando até a 50\% no período 2012.1. Diante desta realidade surge o questionamento sobre a utilização de ferramentas que possam motivar mais os alunos e evitar tamanha retenção, o que levaria a mais profissionais sendo formados, em uma área com demanda muito forte.

\section{Metodologia}

Esta pesquisa utilizou dois métodos: survey e mapeamento sistemático. Ambos configuram-se em uma pesquisa exploratória (Gil, 2008). O survey foi utilizado para identificar os principais conteúdos de POO que os alunos de SI e LCC consideram 
difíceis e o mapeamento sistemático identificou OAs que podem auxiliar o ensino de tais conteúdos.

\subsection{Survey}

Diante da realidade dos altos índices de reprovação expostos na Figura 1 e 2, surgiu à necessidade de descobrir quais são os principais conteúdos do paradigma $\mathrm{OO}$ que os alunos consideram de difícil compreensão.

O questionário online ${ }^{2}$ foi preenchido pelos alunos dos cursos de SI e LCC. O pré-requisito para o preenchimento era que tivessem cursado a disciplina introdutória de programação $\mathrm{OO}$. O formulário é constituído por sete perguntas objetivas e foi respondido por 74 alunos da UFPB - Campus IV.

Uma das perguntas do questionário ( $6^{\circ}$ pergunta) foi sobre os conteúdos considerados pelos alunos como de difícil compreensão. Alguns conteúdos foram sugeridos como opções no formulário, mas os participantes poderiam sugerir outros. Os resultados das respostas estão apresentados na Figura 3.

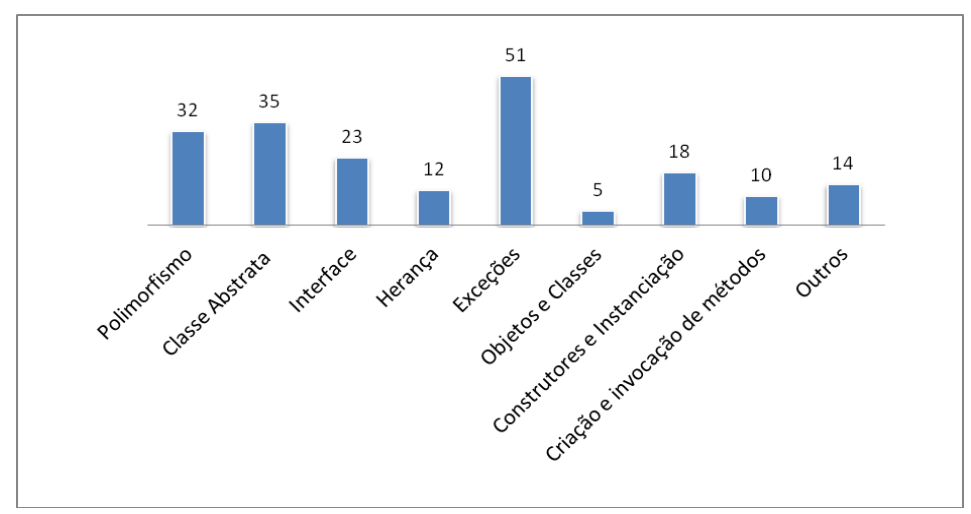

Figura 3 - Conteúdos de POO considerados difíceis pelos alunos de SI e LCC

Observando a Figura 3 percebe-se que o conteúdo considerado mais complexo é "Exceções", com 51 indicações. Logo em seguida está o conteúdo "Classes abstratas", com 35 indicações e "Polimorfismo" com 32 indicações. Portanto, esses três conteúdos são considerados complexos de acordo com a pesquisa realizada e eles foram considerados como prioritários para realizar as análises de conteúdos cobertos pelos OAs selecionados por esse trabalho.

A última pergunta do questionário pedia uma justificativa dos participantes em relação aos conteúdos apontados na questão anterior. A maior parte dos participantes (54\%) disse que sentiram dificuldade em aprender o conteúdo, porém conseguiram dominá-lo posteriormente. Já $30 \%$ dos alunos disseram que ainda não tinham conhecimento sobre os conteúdos apontados por eles como complexos, evidenciando que o aluno não aprendeu de maneira satisfatória e apenas $4 \%$ disseram que não viram os conteúdos que optaram na pesquisa. $12 \%$ dos alunos optaram por outras justificativas que explicitaram como: "Alguns domino", "Aprendi estudando ao longo do tempo, mas não pratico e tenho vaga noção dos conceitos", "Foram ministrados, senti dificuldades e não os domino", "Aprendi o suficiente, não pretendo trabalhar nessa área". Por falta de espaço, não foi possível discutir aqui todas as questões do survey. Todos os resultados estão no resumo das respostas ao questionário online ${ }^{1}$.

\footnotetext{
${ }^{2}$ https://goo.gl/muViIp
} 


\subsection{Mapeamento sistemático}

Para identificar os principais objetos de aprendizagem relacionados ao tema deste trabalho, foi realizado um mapeamento sistemático da literatura. Segundo Kitchenham e Charters (2007) o mapeamento sistemático é um método que fornece uma visão ampla de uma área de pesquisa, e estabelece evidências sobre um determinado tema de forma sistemática.

Portanto, o protocolo utilizado nesta pesquisa foi elaborado com informações baseadas no trabalho de Kitchenham e Charters (2007). Ele está dividido em três fases, a saber: planejamento, condução e documentação. Elas serão detalhadas a seguir.

\subsubsection{Planejamento}

\section{- Questões de pesquisa}

Q1. Quais são os objetos de aprendizagem propostos na literatura para apoiar o ensino de POO?

Q2. Quais conteúdos de OO os objetos de aprendizagem mencionados trabalham?

Q3. Os OAs selecionados são referenciados como OAs pelos trabalhos que os citam?

\section{- Fonte de busca e critérios de inclusão e exclusão}

$O$ engenho de busca automático utilizado na pesquisa dos trabalhos científicos foi a biblioteca digital da ACM (ACM Digital Library ${ }^{3}$ ). Além disso, foi feita uma revisão manual dos artigos do $\mathrm{SBIE}^{4}$ (Simpósio Brasileiro de Informática na Educação). A Tabela 2 mostra os critérios de inclusão e exclusão utilizados para selecionar os trabalhos.

Tabela 2 - Critérios de Inclusão e exclusão

\begin{tabular}{|l|l|}
\hline \multicolumn{1}{|c|}{ Critérios de Inclusão (CI) } & \multicolumn{1}{|c|}{ Critérios de Exclusão (CE) } \\
\hline $\begin{array}{l}\text { CI1 - Foram selecionados os trabalhos que utilizam } \\
\text { Objetos de Aprendizagem para o ensino de OO, mesmo } \\
\text { que os chamem de ferramentas. }\end{array}$ & $\begin{array}{l}\text { CE1 - Não foram incluídos trabalhos focados em } \\
\text { metodologias de ensino de OO utilizando a linguagem } \\
\text { Java. }\end{array}$ \\
\hline $\begin{array}{l}\text { CI2 - Os artigos que apresentaram propostas de ensino } \\
\text { de OO através de IDEs (ambientes integrados de } \\
\text { desenvolvimento) também foram incluídos. }\end{array}$ & $\begin{array}{l}\text { CE2- Foram excluídos os trabalhos que utilizam a } \\
\text { abordagem de programação OO como meio para ensinar } \\
\text { matemática. }\end{array}$ \\
\hline CI3 - Artigos entre 2001 e 2012 & $\begin{array}{l}\text { CE3 - Foram excluídos artigos que não poderiam ser } \\
\text { baixados a partir da rede da UFPB (Por exemplo, alguns } \\
\text { artigos de jornais não estão disponíveis para } \\
\text { universidades públicas). }\end{array}$ \\
\hline
\end{tabular}

\subsubsection{Condução}

\section{- Estratégia de busca e seleção dos trabalhos}

A seleção dos trabalhos foi conduzida por buscas manuais e automáticas. Ambas as estratégias seguiram duas etapas, a saber: (i) leitura do título, resumo (considerando os critérios de inclusão e exclusão); (ii) leitura completa dos trabalhos e extração dos dados.

\footnotetext{
${ }^{3}$ ACM DL: http://dl.acm.org/

${ }^{4}$ SBIE: http://www.br-ie.org/pub/index.php/sbie/issue/archive
} 
Para a busca automática foi elaborada uma string de busca. Esta foi baseada em termos importantes, baseados nas perguntas da pesquisa. O conector OR foi utilizado para combinar os termos alternativos e o conector AND para ligar as palavras chaves. A chave de busca utilizada foi: "teach Object Oriented Programming" or "teach OO Programming" or "learn OO" or "learn Object-Oriented Programming". Na busca manual, procurou-se no título dos trabalhos pelos termos em português.

\section{- Extração dos dados}

Os dados foram extraídos e organizados em uma planilha, contendo os seguintes campos: identificador (EP = Estudo Primário), referência do trabalho e respostas para as questões de pesquisa.

A fase de documentação será descrita na Seção 5, tendo em vista que ela faz parte dos resultados gerados após a síntese dos dados.

\subsubsection{Ameaças à validade}

O protocolo do mapeamento sistemático da literatura seguiu etapas bem definidas. Porém, foram observadas algumas limitações:

- Se a pesquisa aqui proposta for realizada em datas ou usuários distintos, os resultados podem ser outros;

- Caso outras bases de dados venham a ser utilizadas, os trabalhos retornados podem ser distintos dos apresentados nesta pesquisa;

- A string de busca utilizada pode não contemplar todos os sinônimos existentes para os termos identificados, podendo ter sido insuficiente para capturar todos os trabalhos relevantes;

Vale salientar que esta pesquisa utilizou a rede interna da UFPB para capturar os trabalhos disponíveis nas bases de dados.

\section{Resultados e discussão}

No total foram selecionados 07 artigos (completos e resumidos) do SBIE e 09 artigos obtidos através da pesquisa utilizando a biblioteca digital da ACM, considerando a metodologia apresentada no protocolo completo com os critérios de inclusão e exclusão adotados. Ao total então foram selecionados para análise 16 artigos. A lista completa pode ser vista em: https://goo.gl/3vZwAW. A Tabela 3 apresenta com detalhes o quantitativo de artigos encontrados e os que foram selecionados para o mapeamento sistemático, considerando os critérios adotados.

Tabela 3 - Quantitativo dos trabalhos retornados e selecionados

\begin{tabular}{|c|c|c|c|c|c|c|c|c|c|c|c|c|c|c|}
\hline \multicolumn{15}{|c|}{ ACM Digital Library } \\
\hline $\begin{array}{l}\text { Resultados } \\
\text { retornados }\end{array}$ & 2001 & 2002 & 2003 & 2004 & 2005 & 2006 & 2007 & 2008 & 2009 & 2010 & 2011 & 2012 & $\begin{array}{l}\stackrel{n}{0} \\
\stackrel{0}{\Xi} 0 \\
\Xi\end{array}$ & 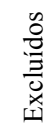 \\
\hline 69 & 0 & 0 & 0 & 1 & 3 & 2 & 0 & 0 & 2 & 0 & 0 & 1 & 9 & 60 \\
\hline \multirow{2}{*}{$\begin{array}{c}\text { Ano de } \\
\text { publicação }\end{array}$} & \multicolumn{14}{|c|}{ Simpósio Brasileiro de Informática na Educação - SBIE } \\
\hline & \multicolumn{3}{|c|}{ Artigos completos } & \multicolumn{2}{|c|}{ Selecionados } & \multicolumn{2}{|c|}{ Excluídos } & \multicolumn{3}{|c|}{ Artigos Resumidos } & \multicolumn{2}{|c|}{ Selecionados } & \multicolumn{2}{|c|}{ Excluídos } \\
\hline 2001 & \multicolumn{3}{|c|}{58} & \multicolumn{2}{|c|}{0} & \multicolumn{2}{|c|}{58} & \multicolumn{3}{|c|}{ Não houve } & \multicolumn{2}{|l|}{-} & \multicolumn{2}{|c|}{-} \\
\hline 2002 & \multicolumn{3}{|c|}{54} & \multicolumn{2}{|c|}{0} & \multicolumn{2}{|c|}{54} & \multicolumn{3}{|c|}{17} & \multicolumn{2}{|l|}{0} & \multicolumn{2}{|c|}{17} \\
\hline 2003 & \multicolumn{3}{|c|}{70} & \multicolumn{2}{|c|}{0} & \multicolumn{2}{|c|}{70} & \multicolumn{3}{|c|}{ Não houve } & \multicolumn{2}{|l|}{-} & \multicolumn{2}{|c|}{ - } \\
\hline
\end{tabular}




\begin{tabular}{|c|c|c|c|c|c|c|}
\hline 2004 & 56 & 0 & 56 & 30 & 1 & 29 \\
\hline 2005 & 63 & 0 & 63 & 10 & 0 & 10 \\
\hline 2006 & 59 & 1 & 58 & 28 & 0 & 28 \\
\hline 2007 & 60 & 1 & 59 & 75 & 0 & 75 \\
\hline 2008 & 80 & 0 & 80 & Não houve & - & - \\
\hline 2009 & 108 & 1 & 107 & 45 & 1 & 44 \\
\hline 2010 & 87 & 0 & 87 & 43 & 0 & 43 \\
\hline 2011 & 91 & 1 & 90 & 47 & 0 & 47 \\
\hline 2012 & 87 & 1 & 86 & 42 & 0 & 42 \\
\hline TOTAL & 873 & $\mathbf{5}$ & 868 & 337 & $\mathbf{2}$ & 335 \\
\hline
\end{tabular}

O quantitativo de artigos publicados por ano sobre Objetos de Aprendizagem para o ensino de OO, na ACM e no SBIE foram bem distribuídos, ficando entre 0 e 4 artigos. O ano com o maior número de publicações incluídas no mapeamento foi em 2009 (4 publicações).

\subsection{Respostas às perguntas de pesquisa}

A primeira pergunta de pesquisa faz o seguinte questionamento: Q1. Quais são os objetos de aprendizagem propostos na literatura para apoiar o ensino de POO?

A Tabela 4 mostra os OAs selecionados. Eles foram categorizados em: IDE, STI, softwares para criar animações e jogos de cartas. Observando a Tabela 4 percebese que as IDEs é a forma mais utilizada para apoiar o ensino de OO, de acordo com o mapeamento sistemático realizado na ACM e SBIE.

Tabela 4 - Categorias de Objetos de aprendizagem selecionados

\begin{tabular}{|l|l|}
\hline \multicolumn{1}{|c|}{ Categorias } & \multicolumn{1}{c|}{ Objetos de aprendizagem } \\
\hline $\begin{array}{l}\text { IDE (Integrated Development Environment), é um } \\
\text { software utilizado para o desenvolvimento de outros } \\
\text { softwares. }\end{array}$ & BlueJ, JavaTool, Object Editor, DrJava e Greenfoot. \\
\hline Sistema Tutores Inteligentes (STI) & CIMEL ITS, PROGTUTOR e AFM. \\
\hline $\begin{array}{l}\text { Ferramentas que utilizam metodologias diferenciadas } \\
\text { de ensino. }\end{array}$ & iVProg, OntoRevPro e RAPTOR e Páginas na WEB. \\
\hline Softwares para criar animações & Alice e Jeliot \\
\hline Jogos de cartas & CRC-cards e Smalltalk Card Game (SCG) \\
\hline
\end{tabular}

A segunda pergunta de pesquisa faz o seguinte questionamento: Q2. Quais conteúdos de $\mathrm{OO}$ os objetos de aprendizagem mencionados trabalham?

A Tabela 5 sugere os OAs que podem auxiliar o ensino de alguns conteúdos de POO, considerando os conteúdos apontados pelos alunos como difíceis (eg. exceções, polimorfismo e classe abstrata). As seguintes ferramentas são indicadas para auxiliar no ensino desses conteúdos: BlueJ, Object Editor, Páginas Web e RAPTOR. Vale ressaltar que o Alice, apesar de ser um software que produz animações, se enquadra no conceito de OA, porque ele tem fins pedagógicos e já é utilizado em várias instituições de ensino para fins pedagógicos (Barros et al, 2012). 
Tabela 5 - OAs que cobrem assuntos considerados difíceis pelos alunos de LCC e SI

\begin{tabular}{|c|c|l|}
\hline Conteúdo & $\begin{array}{c}\text { Percentual de alunos que } \\
\text { consideraram esse } \\
\text { conteúdo difícil }\end{array}$ & \multicolumn{1}{|c|}{ OAs Selecionados } \\
\hline Exceções & $51 / 74(68,9 \%)$ & - \\
\hline Classes Abstratas & $35 / 74(47,3 \%)$ & BlueJ \\
\hline Polimorfismo & $32 / 74(43,2 \%)$ & BlueJ, Object Editor, Páginas Web e RAPTOR \\
\hline Interface & $23 / 74(31,1 \%)$ & Páginas Web e RAPTOR \\
\hline $\begin{array}{c}\text { Construtores e } \\
\text { Instanciação }\end{array}$ & $18 / 74(24,3 \%)$ & Object Editor e BlueJ \\
\hline Herança & $12 / 74(16,21 \%)$ & BlueJ e RAPTOR \\
\hline $\begin{array}{c}\text { Criação e invocação } \\
\text { de métodos }\end{array}$ & $10 / 74(13,51 \%)$ & BlueJ, Jeliot, Object Editor, Alice, ivProg e Javatool. \\
\hline $\begin{array}{c}\text { Objetos e Classes } \\
5 / 74(6,75 \%)\end{array}$ & $\begin{array}{l}\text { Greenfoot, BlueJ, AFM, CRC- Cards, AFM, Smaltalk } \\
\text { Card, iVProg, CRC-cards, Alice, PROGTUTOR, } \\
\text { OntoRevProv e Javatool }\end{array}$ \\
\hline
\end{tabular}

Existem vários trabalhos que relatam os benefícios trazidos com o uso de OAs no ensino de POO. O trabalho de Bittencourt et al (2013) apresenta a experiência de oficinas de aprendizagem, duas delas tiveram o foco em noções básicas de POO e Java utilizando o Greenfoot e BlueJ. Os resultados mostram que propor um ambiente lúdico de aprendizagem trouxe motivação para os alunos e a aprendizagem se tornou mais significante, pois os alunos aprendem fazendo (construção de jogos e diagramas UML).

A terceira pergunta de pesquisa faz o seguinte questionamento: Q3. Os OAs selecionados são referenciados como OAs pelos trabalhos que os citam?

Durante a leitura dos artigos selecionados, percebe-se que os Objetos de Aprendizagem citados pelos trabalhos não são referenciados como OAs, pois não são usados nos trabalhos os termos: "Objeto de aprendizagem" ou em inglês "Learning object" ao falar neles. Porém, este trabalho os considera OAs devido à variedade de recursos apresentados pela sua interface e por trabalharem algum conceito de uma área do conhecimento. Algo interessante a observar é que os OAs selecionados pelo estudo podem ser utilizados para construir diferentes materiais instrucionais, como vídeos, considerando os conteúdos identificados pelos alunos, como forma de apoiar no ensinoaprendizagem de $\mathrm{OO}$, o que pode ser uma importante contribuição para a área no sentido de motivar mais os alunos e deixar mais claros conceitos abstratos.

\section{Considerações finais}

Considerando os resultados apresentados neste trabalho, acredita-se que há vários OAs para apoiar o ensino de $\mathrm{OO}$ e que podem ser utilizados para trabalhar alguns conteúdos de $\mathrm{OO}$ considerados difíceis segundo o survey realizado com os alunos de LCC e SI. Porém, nem sempre eles são referenciados na literatura como Objetos de Aprendizagem, embora possam ser consideradas como tais já que auxiliam o ensino de conteúdos de uma área do conhecimento (computação) e que tudo dependerá da forma como forem utilizados. O estudo mostrado também dá uma ideia de conteúdos a explorar no projeto e construção de novos OAs para apoiar o ensino de $\mathrm{OO}$ e reforça a necessidade de OAs para tratar do conteúdo exceções, apontado por grande parte dos alunos como um conteúdo difícil.

Pretende-se como trabalho futuro estender a revisão sistemática da literatura em outras bases de dados, com o propósito de selecionar uma variedade maior de OAs para que professores de POO tenham um leque de possibilidades, tornando assim o ambiente de ensino motivador, além de poderem construir materiais instrucionais relevantes com esses OAs, como vídeos com bons exemplos de OO animados com essas ferramentas. 
Outra proposta é realizar oficinas de aprendizagem semelhantes ao trabalho de Bittencourt et al (2013), com as turmas de LCC e SI da UFPB - Campus VI, com o objetivo de propor um ambiente lúdico e motivar os alunos durante o processo de ensino e aprendizagem.

Além disso, analisando com mais detalhes os OAs identificados neste trabalho, os assuntos que cobrem e por não serem citados como objetos de aprendizagem pelos trabalhos publicados, propõe-se como trabalho futuro o projeto e a criação de um OA que cubra alguns conteúdos difíceis pouco cobertos (como exceções) e que atenda a critérios de avaliação de OAs, como os propostos por Reategui et al. (2010).

\section{Referências bibliográficas}

AURELIANO. V. C. O; TEDESCO. P. C. A. R. Ensino-aprendizagem de Programação para Iniciantes: uma Revisão Sistemática da Literatura focada no SBIE e WIE. In: Anais do Simpósio Brasileiro de Informática na Educação. Vol. 23. № 1. 2012.

BARROS. E. A. R; GRINKRAUT. M.L; ZAMBONI. L.C; TSAN HU. O.R. PAMBOUKIAN. S.V.D; TCHEMRA. A.H; CRAVEIRO. M. V. Alice: uso do software no processo educacional junto aos cursos de engenharia. COBENGE - XL Congresso brasileiro de educação e engenharia. Belém - Pará, 2012.

BITTENCOURT, R. A.; ROCHA, A. S.; SANTANA, B. L.; SANTANA, C. S.; CARNEIRO, D. A.; BORGES, G. A.; CHALEGRE. H.S; SILVA. J. F. J; SANTOS. J. N. J; SILVA. L.A; ANDRADE, P. H.M.O. Aprendizagem de Programação Através de Ambientes Lúdicos em um Curso de Engenharia de Computação: Uma Primeira Incursão. In: Anais do Workshop sobre Educação em Computação, 2013.

FLÔRES. M. L. P.; TAROUCO. L. M. R. Diferentes tipos de objetos para dar suporte a aprendizagem. RENOTE - Revista Novas Tecnologias na Educação, V. 6 N $^{\mathrm{o}}$ 1, Julho, 2008 .

GIL. A. C - Como elaborar projetos de pesquisa. 6. ed. - São Paulo : Atlas, 2008

KITCHENHAM, B., CHARTERS S. Guidelines for performing Systematic Literature Reviews in Software Engineering. Vol 2.3 Technical Report, EBSE-2007-01, Software Engineering Group, School of Computer Science and Mathematics, Keele University, 2007

MARCOLINO, A.; BARBOSA, E. F. Softwares Educacionais para o Ensino de Programação: Um Mapeamento Sistemático. In: Anais do Simpósio Brasileiro de Informática na Educação, Vol. 26, Nº 1, p. 190. 2015

MEDEIROS, T. J.; SILVA, T. R.; ARANHA, E. H. S. Ensino de programação utilizando jogos digitais: uma revisão sistemática da literatura. RENOTE - Revista Novas Tecnologias na Educação, v. 11, n. 3, 2013

RAABE, A. L. A.; SILVA, J. M. C. Um Ambiente para Atendimento as Dificuldades de Aprendizagem de Algoritmos. In: XXV Congresso da Sociedade Brasileira de Computação. São Leopoldo/RS. 2005.

REATEGUI. E.; BOFF, E.; FINCO, M. D. Proposta de Diretrizes para Avaliação de Objetos de Aprendizagem Considerando Aspectos Pedagógicos e Técnicos. RENOTE Revista Novas Tecnologias na Educação, v. 8, n. 3, 2010

SILVA, T. R.; MEDEIROS, T. J.; ARANHA, E. H. S. Jogos Digitais para Ensino e Aprendizagem de Programação: uma Revisão Sistemática da Literatura. In: Anais do Simpósio Brasileiro de Informática na Educação, Vol. 25, No 1, p. 692-701. 2014 WILEY. D. A. Connecting learning objects to instructional design theory: a definition, a metaphor, and a taxonomy. The Instructional Use of Learning Objects: on-line version. 2000. In: WILEY, D. A. (Ed.). Disponível em: http://wesrac.usc.edu/wired/bldg7 file/wiley.pdf. Acesso em 25 out. 2015. 